\title{
The Role of Polarization Diversity for MIMO Systems Under Rayleigh-Fading Environments
}

\author{
Juan F. Valenzuela-Valdés, Miguel A. García-Fernández, Antonio M. Martínez-González, and \\ David Sánchez-Hernández, Senior Member, IEEE
}

\begin{abstract}
Polarization diversity techniques have not received as much attention as others due to the significant difference in mean signal level between copolarized and cross-polarized branches when one polarization is transmitted. However, multiple-input-multiple-output (MIMO) systems where the multipath fading is only partially correlated could use polarization diversity to provide a high diversity gain. Hence there is a need to fully understand the role of true polarization diversity in such systems. In this letter, progressive (true) polarization diversity performance for $3 \times 3$ MIMO systems under Rayleigh-fading environments is evaluated through simulations and measurements. True polarization diversity was found to be as significant as spatial diversity at improving diversity gain, and hence MIMO system capacity.
\end{abstract}

Index Terms-Diversity gain, multiple-input-multiple-output (MIMO) systems, polarization diversity.

\section{INTRODUCTION}

$\mathbf{P}$ OLARIZATION diversity, early applied to HF, radar, and imaging systems, has demonstrated its potential for improving the capacity of wireless communications systems, despite some disappointing premature predictions [1]. The improvement is typically granted by an additional decorrelated channel provided by a polarization state made orthogonal to the existing one, usually at the transmitting end $(\mathrm{T}=2)$. A randomly orientated linearly-polarized antenna is also typically used at the receiver $(\mathrm{R}=1)$ for evaluating polarization diversity. Consequently, the cross-polarization discrimination (XPD) factor is the usual evaluating parameter, with low correlation coefficients being achieved even in NLOS situations [2]. Due to the significant difference in mean received signal level between copolarized and cross-polarized branches when one polarization is transmitted, considerably more attention has been paid to spatial diversity. The (de)coupling effect between different polarizations is a complex mechanism to be simulated, which has also limited true polarization diversity research. Yet, since at least $20 \lambda$ horizontal and $15 \lambda$ vertical separation distances are required for efficient spatial outdoor diversity in practice, polarization diversity has recently gained attraction. The use of vector antennas which can respond to more than one component and/or

\footnotetext{
Manuscript received September 5, 2006; revised November 15, 2006. This work was supported in part by Fundación Séneca, the R\&D coordinating unit of the Autonomous Region of Murcia (Spain) under project reference 2I05SU0033. The authors wish to thank Bluetest $A B$ for its generous educational discounting.

The authors are with the Departamento de Tecnologías de la Información y Comunicaciones, Universidad Politécnica de Cartagena, Cartagena E-30202 Spain (e-mail: david.sanchez@upct.es; http://www.gimre.upct.es).

Digital Object Identifier 10.1109/LAWP.2006.889552
}

polarization states of the EM field through colocation instead of more voluminous spatially separated arrays can provide equivalent channel capacity increase [3]. The $\pm 45^{\circ}$ slanted polarization diversity combination was found to perform just as good as the spatial polarization diversity with two elements, and commercial systems like GSM and UMTS soon switched to this new technique. Some combinations of two-branch polarization and spatial diversity have also been reported [4]. Recently, a triaxial combination of polarization and pattern diversity has also been proposed [5], with some contradicting results.

In mobile communications scenarios, however, multiple scattering may not be sufficient for a given polarization to decouple half its power into the orthogonal polarization [6]. Channel behavior is therefore different for different polarization states [6] and fading cross-correlations may even increase the ergodic capacity beyond the case of independent channels [7]. Hence, diversity research results based upon orthogonal polarization states cannot be generalized to arbitrary (true) polarization states for more general TxR MIMO systems. On the other hand, MIMO systems do not require perfectly uncorrelated signals to perform near full capacity, and more than two orthogonal polarizations could be used efficiently [8]. Yet, the full potential of true polarization diversity where any polarization state could be employed is not fully addressed in the literature and requires more research [9].

In this letter, the role of true polarization diversity for MIMO systems under Rayleigh-fading scenarios is investigated through simulations and measurements in a reverberation chamber. Since true polarization diversity can be successfully combined to other diversity schemes, which could be limited due to spatial or coherent bandwidth restrictions, the results presented in this paper are of particular importance for future diversity-combined MIMO systems.

\section{Simulation AND MeAsurements}

The multipath environment can be generated artificially in a reverberation chamber fed by wall-mounted antennas, which thereby provides a statistically repeatable laboratory-produced environment for characterizing mobile terminals and antennas, well described in the literature. The processed S-parameters represent estimates of the matrix $\mathrm{H}$ of multipath communication channels set up between the wall antennas and the MIMO array inside the chamber. Apparent diversity gain for the selection combining (SC) or maximal ratio combining (MRC) techniques is obtained from the processed S-parameters by evaluating the 


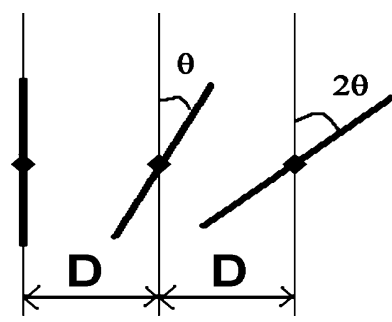

Fig. 1. Geometries of analyzed MIMO scenarios.

cumulative probability distributions of the measured channel samples received at each MIMO array antenna

$$
h_{m n}=\frac{S_{m n} \sqrt{e_{\text {ref }}\left(1-\left|\bar{S}_{n n}\right|^{2}\right)}}{T_{\text {ref }}}
$$

where $m$ is the index for the transmitting antenna, $n$ is the index for the receiving antenna, $T_{\text {ref }}$ is the net chamber transfer function for the reference antenna and $e_{\text {ref }}$ is its radiation efficiency. The effective diversity gain is the increase from the reference level to the combined signal that is observed at the $1 \%$ probability level.

All measurements illustrated in this paper have been performed with the $\mathrm{RC} 800$ reverberation chamber by Bluetest $\mathrm{AB}$, with dimensions of $0.8 \mathrm{~m} \times 1 \mathrm{~m} \times 0.6 \mathrm{~m}$, three wall-mounted transmitting antennas, 25 platform stirring positions, two mechanical stirrers with 15 different positions for each platform position and $100 \mathrm{MHz}$ frequency stirring. Measurements were performed at $900 \mathrm{MHz}$ and 3 dipoles were used as receiving MIMO array antennas following the setup depicted in Fig. 1.

SC diversity gain increments were evaluated by rotating a certain angle an antenna with regard to a contiguous one, namely, by applying a progressive angular separation between elements, and compared to measured SC spatial diversity performance and their simulated MIMO Rayleigh-fading channels counterparts. Polarization diversity was applied at both transmitting and receiving ends.

Fig. 2 illustrates a comparison between spatial and polarization diversity for 20 different measured $3 \times 3$ MIMO systems, showing wavelength-normalized spatial separation $\mathrm{D}(\mathrm{d} / \lambda)$ and angular separation $\mathrm{d} \theta\left(^{\circ}\right)$ versus measured diversity gain with $T=3$ and $R=3$. From this figure it is clearly observed that an equivalence scenario is obtained for the two diversity techniques. As an example, a spatial separation of $\sim 0.12 \lambda$ is equivalent to an angular separation of $\sim 36^{\circ}$ for the $3 \times 3$ MIMO systems under evaluation.

In order to evaluate the full potential of combined-diversity techniques, SC diversity gain measurements were performed for three receiving dipoles separated by both an angular and a spatial separation. Fig. 3 shows the measured diversity gain versus angular separation $\mathrm{d} \theta$ with spatial separation $\mathrm{D}$ as a parameter. As expected, the combination of both spatial and polarization diversity provided increased diversity gain with only three elements in the array. When the spatial separation is large, the angular separation can hardly improve the diversity gain. Similarly, when the angular separation is large, the spatial separation can barely improve the diversity gain. This suggests that a

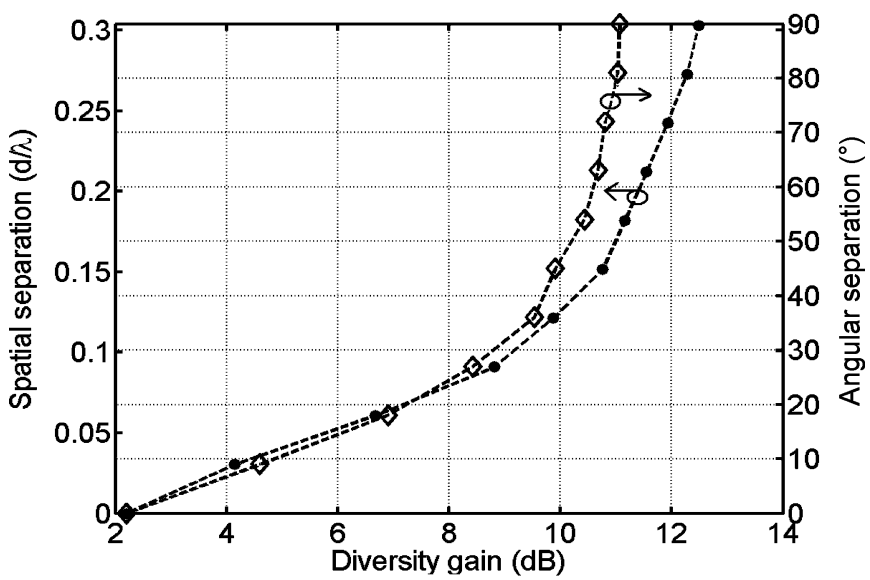

Fig. 2. Spatial separation $\mathrm{D}(\mathrm{d} / \lambda)$ and angular separation $d \theta\left({ }^{\circ}\right)$ versus measured diversity gain with $\mathrm{T}=3, \mathrm{R}=3 .---\diamond----$ Angular separation $\left.{ }^{\circ}\right)$ with $\mathrm{D}=0 ;-\cdots \bullet---$ Spatial separation $(\mathrm{d} / \lambda)$ with $d \theta=0$.

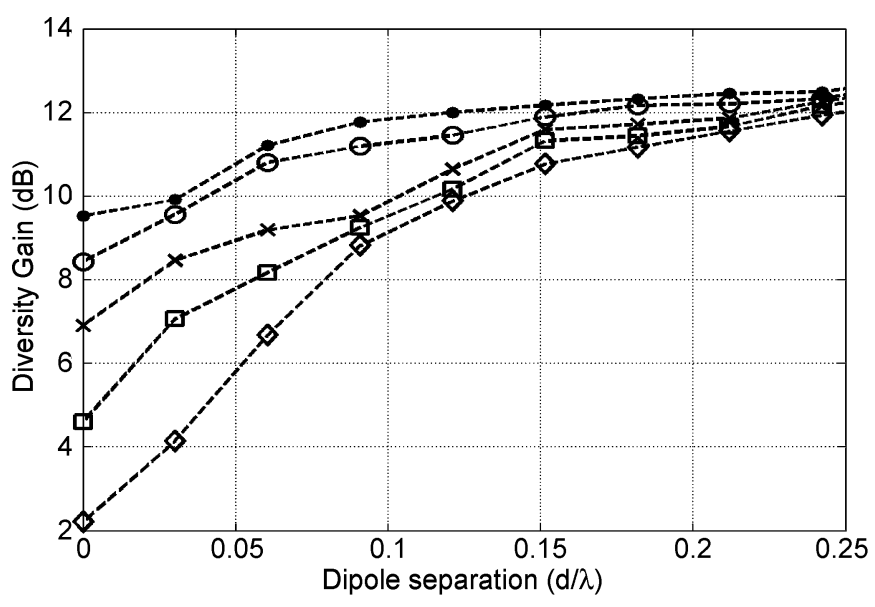

Fig. 3. Measured diversity gain versus Dipole separation $D(d / \lambda)$ with angular separation $\mathrm{d} \theta\left({ }^{\circ}\right)$ as a parameter and $\mathrm{T}=3, \mathrm{R}=3 . \cdots \diamond-\cdots \mathrm{d} \theta=0$; $\mathrm{d} \theta=36$.

good combination of the two diversity techniques with both low spatial and angular separation values represents the most efficient method for optimum diversity performance for the same reduced available volume.

\section{CONCLUSION}

The full potential of spatial and true polarization diversity obtained in a set of rotational linearly polarized elements in both transmitting and receiving ends of $3 \times$ MIMO systems has been investigated. Measured results have demonstrated that spatial diversity can provide considerable gain to a MIMO system. We have also demonstrated that polarization diversity is equally important and particularly interesting for volume-limited schemes where the full potential of spatial diversity techniques cannot be exploited.

Future research should concentrate on more general TxR MIMO systems, system capacity and diverse fading environments to investigate the full capabilities of rotational polarization diversity schemes and combined-diversity methods. 


\section{REFERENCES}

[1] M. S. Smith and L. Neal, "A comparison of polarization and space diversity for indoor propagation at $900 \mathrm{MHz}$," in Proc. 2nd Int. Conf. Universal Personal Communications: Gateway to 21st Century, vol. 1, Oct. 1993, pp. 74-78.

[2] J. J. A. Lempiainen and J. K. Laiho-Steffens, "The performance of polarization diversity schemes at a base station in small/micro cells at 1800 MHz," IEEE Trans. Veh. Technol., vol. 47, no. 3, pp. 1087-1092, Aug. 1998.

[3] A. S. Konanur, K. Gosalia, S. H. Krishnamurthy, B. Hughes, and G. Lazzi, "Increasing wireless channel capacity through MIMO systems employing co-located antennas," IEEE Trans. Microw. Theory Techn., vol. 53, no. 6, pp. 1837-1844, Jun. 2005.

[4] K. Cho, T. Hori, and K. Kagoshima, "Effectiveness of four-branch height and polarization diversity configuration for street microcell," IEEE Trans. Antennas Propagat., vol. 6, no. 6, pp. 776-781, Jun. 1998.
[5] M. C. Tumbuka and D. J. Edwards, "Investigation of tri-polarized MIMO technique," Electron. Lett., vol. 41, no. 3, pp. 137-138, Feb. 2005.

[6] R. G. Vaughan, "Polarization diversity in mobile communications," IEEE Trans. Veh. Technol., vol. 39, pp. 177-186, Aug. 1990.

[7] C. Oestges and A. J. Paulraj, "Beneficial impact of channel correlations on MIMO capacity," Electron. Lett., vol. 40, no. 10, pp. 606-608, May 2004.

[8] C. Waldschmidt, C. Kuhnert, T. Fügen, and W. Wiesbeck, "Measurements and simulations of compact MIMO-systems based on polarization diversity," in Proc. IEEE Topical Conf. Wireless Commun. Technol., 2003, pp. 284-285.

[9] R. M. Narayanan, K. Atanassov, V. Stoiljkovic, and G. R. Kadambi, "Polarization diversity measurements and analysis for antenna configurations at $1800 \mathrm{MHz}$," IEEE Trans. Antennas Propagat., vol. 52, no. 7, pp. 1795-1810, Jul. 2004. 